Voix et Images

voixetimages

\title{
Entretien avec Marie-Claire Blais
}

\section{Nathalie Roy et Anne Élaine Cliche}

Volume 37, numéro 1 (109), automne 2011

Marie-Claire Blais

URI : https://id.erudit.org/iderudit/1006458ar

DOI : https://doi.org/10.7202/1006458ar

Aller au sommaire du numéro

Éditeur(s)

Université du Québec à Montréal

ISSN

0318-9201 (imprimé)

1705-933X (numérique)

Découvrir la revue

Citer ce document

Roy, N. \& Cliche, A. É. (2011). Entretien avec Marie-Claire Blais. Voix et Images, 37(1), 15-25. https://doi.org/10.7202/1006458ar d'utilisation que vous pouvez consulter en ligne.

https://apropos.erudit.org/fr/usagers/politique-dutilisation/ 
VoIX ET IMAGES Ce numéro de Voix et Images porte sur les stratégies narratives et poétiques et, plus largement, sur les questions liées à l'esthétique particulière de vos romans. Dans le cadre de cet entretien, nous aimerions nous arrêter à ces dimensions de votre écriture, quitte à nous éloigner un peu d'un genre de questions qu'on vous pose de façon insistante, au sujet des enjeux moraux ou politiques de votre écriture. Nous voulons partir du principe que, pour saisir dans vos œuvres le regard porté sur le monde, il est essentiel de poser la question de la forme. On ne peut séparer la forme du sens, comme le disait déjà Flaubert, pour qui le style, on s'en souvient, est une «manière absolue de voir les choses ${ }^{2} »$. Dans cet esprit, nous voudrions vous interroger d'abord sur la manière dont la musique, la peinture et d'autres textes littéraires informent votre démarche d'écriture.

Ainsi, nous aimerions que vous vous exprimiez sur le rôle que joue la musique dans la composition de vos textes, dans leur rythme, leurs variations d'intensité, leurs nuances, leurs jeux polyphoniques, etc. Votre prose a sa musicalité propre et on peut avoir l'impression en vous lisant d'entendre une pièce classique interprétée par un orchestre. Avez-vous en tête des œuvres particulières lorsque vous écrivez? Si oui, déterminent-elles d'une quelconque façon la structure de vos textes? Dans les romans du cycle Soifs, certaines œuvres de musique classique sont évoquées à plusieurs reprises. Par exemple, dans Augustino et le chœur de la destruction, il est très souvent question du Réveil des oiseaux d'Olivier Messiaen et du Requiem de guerre de Benjamin Britten. On voit aisément en quoi, d'un point de vue thématique, ces œuvres peuvent agir comme une clé de lecture, mais peut-on penser que les structures et les mouvements de ces compositions précises informent votre écriture du roman?

1 Cet entretien accordé par Marie-Claire Blais à Nathalie Roy a été mené par courrier électronique entre le 17 juillet et le 2 septembre 2010. Les questions ont été formulées par Nathalie Roy et par Anne Élaine Cliche. Nous avons choisi de nous en tenir à une seule question par message, afin de pouvoir nous adapter aux tournures que prenait l'échange. 2 Lettre du 16 janvier 1852 à Louise Colet, Gustave Flaubert, Euvres complètes. Correspondance, deuxième série, Paris, Louis Conard, 1926, p. 346. 
MARIE-CLAIRE BLAIS Comme vous l'avez remarqué, les compositions musicales sont souvent liées à l'atmosphère immédiate du livre, comme si la musique pouvait porter les mots, les transcender, par une voix nouvelle, plus lumineuse et plus souple que les mots, qui doivent passer par la douleur et la lourdeur de ce qu'ils ont à exprimer. Dans Augustino et le chœur de la destruction, il y a la lumière de Messiaen (sa foi en la nature, son amour du chant des oiseaux, sa confiance en une terre encore habitable et belle), qui affronte en même temps les pensées d'Augustino sur la guerre et la violence dans le monde, Augustino dont la conscience est plus près du requiem de Britten que de l'enchantement de Messiaen, mais dont l'âme est emportée par les deux musiques, car il est très jeune et est ouvert à ce qui lui apporte de l'espérance, comme il peut comprendre aussi la tristesse de Britten, dans ce requiem, et sa dénonciation de la violence. Augustino sait que sa vie est aussi précaire que celle des jeunes soldats que décrit Britten dans son requiem, que c'est là la tragédie de l'époque qui est la sienne; toutefois, il est séduit par cette luminosité de Messiaen, même s'il ne croit en rien de ces promesses de la cité céleste qu'évoque le musicien. Il aime être séduit, enchanté.

VoIX ET IMAGES Votre remarque sur le rapport entre les compositions musicales et l'atmosphère immédiate du livre est très intéressante, notamment quand vous dites que $c^{\prime}$ est «comme si la musique pouvait porter les mots, les transcender, par une voix nouvelle, plus lumineuse et plus souple». Nous comptons revenir un peu plus loin sur cette dimension de votre écriture, et sur ce qui caractérise le lyrisme propre à vos textes, cette possibilité de "transcender» les mots. Vous parlez du fait que, dans le troisième volet du cycle Soifs, l'âme d'Augustino est emportée par les deux musiques, celle de Britten et celle de Messiaen, bien que sa conscience soit plus près du requiem. Dans Naissance de Rebecca à l'ère des tourments, le personnage semble encore davantage habité par cette hantise de la violence et de la destruction. Mère tâche d'opposer à ses propos sombres la luminosité du monde tel que le représente Messiaen, mais on a l'impression que le désespoir menace à tout moment d'emporter le jeune écrivain, devenu célèbre pour sa Lettre à des jeunes gens sans avenir. Dans Mai au bal des prédateurs, on apprend que le personnage séjourne à Calcutta, mais on ne sait trop ce qu'il est allé y faire, bien qu'on suppose qu'il y est à des fins humanitaires. À moins que ce soit pour mieux témoigner de la misère qui y sévit. Pouvez-vous nous parler un peu de l'évolution de ce personnage dont la sensibilité d'écrivain est si importante - comme vous l'avez déjà dit vous-même dans un autre entretien ${ }^{3}-$ dans les romans du cycle?

MARIE-CLAIRE BLAIS On verra dans le sixième livre de Soifs qu'Augustino séjournait à Calcutta comme travailleur humanitaire dans un orphelinat, mais qu'il était aussi là pour écrire et être un témoin de son temps, bien qu'il doute fort de ce rôle - c'est aussi un jeune homme comme un autre qui se cherche beaucoup à travers l'écriture. C'est un garçon d'aujourd'hui en quête de spiritualité, et qui ira de recherche spirituelle en recherche spirituelle, un chercheur qui devra acquérir comme malgré lui un certain bonheur de vivre qu'il n'éprouve pas dans les livres précédents. Cette lumière

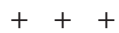

3 Marie Hélène Poitras, «La condition humaine», Voir, Montréal, 24 mars 2005, p. 49. 
lui viendra aussi des enseignements de sa mère, qui l'a beaucoup marqué. Mais ses crises de doutes quant à l'avenir de sa génération seront toujours profondes. Samuel, le frère aîné d'Augustino, est un visionnaire : il sait pressentir toutes les catastrophes et les décrit presque esthétiquement dans ses chorégraphies, danses, etc., mais il y a toujours en lui cet espoir qui est celui de l'artiste dans ses créations. Il s'acharne à décrire ce qu'il ressent (voir sa récente chorégraphie de Venise en une nuit, sous le déluge, dans Mai au bal des prédateurs) et ne désespère jamais, il est un peu comme Cocteau, toujours gracieux, emporté, poétique, et a du mal avec les restrictions de la vie quotidienne et l'enfermement familial. Les deux frères sont très différents, mais partagent peut-être un semblable idéal de transformation de l'humanité.

VOIX ET IMAGES Il y a dans le lyrisme propre à vos textes comme une manière d'interpeller les sens, pour transcender certes la lourdeur et la douleur des mots et de ce qu'ils doivent exprimer, comme vous le disiez, mais peut-être aussi pour dépasser le caractère forcément abstrait et linéaire du langage. En lisant, on est appelé à entendre, à voir, à sentir même. De la même manière que la musique, la peinture joue un rôle structurel dans vos romans. La dimension visuelle de vos textes vous amène en quelque sorte à déjouer la linéarité et l'abstraction des mots pour faire voir. La critique a souligné l'importance du Cri d'Edvard Munch, en tant que motif qui inspire la forme narrative, dans Le sourd dans la ville. Y a-t-il dans les romans du cycle Soifs de semblables tableaux qui informent structurellement la composition des textes?

MARIE-CLAIRE BLAIS Ces tableaux dont vous parlez font partie de la structure de plusieurs livres, avant le cycle de Soifs. Ainsi, il est longtemps question d'un tableau de Boudin dans Visions d'Anna, lequel repose l'âme agitée d'Anna pendant son périple ou errance au Mexique; on reconnaît déjà un tableau de Dürer, Melancholia, dans les Manuscrits de Pauline Archange, représentant le labeur de l'artiste dans son atelier, parmi ses animaux domestiques, un chat, un chien, si je me souviens bien. Le premier ami de Pauline devenue écrivain est André Chevreux, qui est perçu comme l'ange boudeur représenté par Dürer dans son tableau: fils de boucher, André souhaite comme Pauline échapper à son destin par l'écriture ou l'art, et pourtant, comme l'ange, il est bien rivé aux nécessités de la terre. L'œuvre de Munch revient sans cesse dans Le sourd dans la ville, il me semble, plusieurs fois, avec l'évocation aussi du si beau portrait de la jeune sœur du peintre à l'agonie - dans son film Le sourd dans la ville, Mireille Dansereau a accordé beaucoup de place à la présence des tableaux, de façon très subtile. Dans Mai au bal des prédateurs, Yinn voit soudain un tableau de Goya, lorsque défile dans les rues le cortège des sidéens (africains ou américains).

VoIX ET IMAGES En effet, la peinture occupe une place essentielle dans vos textes. Toutefois, on a le sentiment, en lisant votre réponse, que son rôle se joue davantage sur le plan thématique que sur les plans matériel ou esthétique. L'omniprésence des tableaux, gravures, sculptures, etc., donne une profondeur de champ à l'expérience des personnages, l'universalise en quelque sorte. Mais, selon nous, elle contribue aussi à donner à votre prose sa facture presque visuelle. Votre écriture a un côté "plastique » tout comme elle a une dimension musicale. À vrai dire, il semble que vous développez dans les livres de Soifs une véritable architectonique narrative, grâce à laquelle les romans ont tout à la fois la forme du récit, la musicalité de la symphonie et la géométrie du tableau (par l'insistance sur des couleurs, des objets visuels). Nous nous 
interrogeons sur les éléments qui entrent dans l'élaboration de la structure de votre prose. Vous avez évoqué dans un entretien récent, à l'occasion de la sortie de Mai au bal des prédateurs, le long temps de préparation qui précède le passage à l'écriture. Pouvez-vous nous parler un peu de ce travail d'élaboration, de la manière dont vous cherchez à penser cette structure?

MARIE-CLAIRE BLAIS Pour chaque écrivain, ce travail d'élaboration avant l'écriture d'un livre est variable, mais chacun sans doute est conscient de la difficulté de cette étape : on ne peut imaginer l'œuvre de Robert Lalonde, de Marguerite Yourcenar, sans une réflexion profonde soutenant le souffle de ces livres, leur qualité littéraire immense ; on sent combien les personnages ont été analysés, étudiés, à fond, et en détail, dans leurs méandres les plus secrets. Souvent l'artiste se sert de sa propre expérience, ou de celle des autres qu'il ne cesse de chercher à comprendre, mais c'est sa sensibilité dans toute son ouverture qui fera le travail d'approche et d'humanisation. Pendant des années, des mois, cet outil le plus vulnérable, la sensibilité de l'artiste, de l'écrivain, s'aiguisera à une activité très forte et dérangeante de compréhension et d'empathie, envers ces êtres (non plus des personnages) qu'il décrira dans ses livres et qui pour lui, ou elle, deviennent une représentation d'une humanité toujours changeante. Je pense aussi que l'écrivain devient peu à peu ceux qu'il décrit à force d'être près d'eux. C'est l'aspect le plus passionnant de l'écriture, il me semble, de pouvoir tout saisir à la fois, sans jugement moral, de ce qui se passe chez l'autre. Mais cela prend beaucoup de temps, c'est un envahissement sublime, mais qui exige beaucoup de l'écrivain, comme engagement personnel - on peut voir, dans le cas de Kafka, combien ses personnages ont eu prise sur lui, sur sa santé, d'autant plus que Kafka, lorsqu'il écrivait Le procès, La colonie pénitentiaire, connaissait le ton prophétique de son œuvre, décrivait l'avenir comme il le deviendrait avec l'oppression historique qui suivrait.

VoIX ET IMAGES Cette sensibilité d'artiste est certainement nécessaire à tout écrivain. Mais nous parlons plutôt de l'élaboration formelle. Ce qui s'est mis en place avec Soifs n'est pas du même ordre que la narration des romans précédents. Nous aimerions beaucoup que vous nous entreteniez de la forme, du style, du travail du langage, de ce qui fait le propre de votre écriture, en somme, qui ne ressemble à aucune autre.

MARIE-CLAIRE BLAIS Ce qui était plus difficile peut-être pour l'écriture de Soifs, c'était de devoir assimiler tous les livres liés au premier livre à la fois, connaître la destinée des personnages à travers le temps. C'est en même temps très fascinant, cette évolution dans le temps. Ainsi l'évolution de Mère, de Samuel, des uns et des autres qui se modifient réellement, cela exigeait une écriture très vive, leste, et la visibilité d'un monde très moderne où toujours tout frémit et se renouvelle, où les événements, les drames politiques et sociaux ne cessent de se bousculer les uns et les autres, comme pour nous, dans nos vies; d'abord femme bourgeoise, généreuse mais très bourgeoise, Mère se transforme de livre en livre, au contact de tous ceux, très éprouvés, qu'elle accueille dans sa maison, au contact de sa fille qu'elle adore; la Mère de Soifs a bien grandi lorsqu' on la retrouve dans Mai au bal des prédateurs. De même chacun des personnages de cette vaste famille a pu se transformer au contact de Mère, de même Renata et son mari, Claude, se transforment dans leurs défis communs face à l'injustice, tandis qu'au tout début, dans le livre Soifs, ils sont davantage préoccupés par l'aspect passionnel de leur vie. 
VoIX ET IMAGES Il est apparemment peu intéressant (ou difficile) pour vous de parler de la forme et des voix au sens scriptural. Ce que vous construisez est pourtant très harmonieux et musical, et implique une écriture ciselée, une prose proche de la poésie. Vous accordez à chaque voix une sorte de phrasé mélodique, vous multipliez les dédoublements et vous juxtaposez au temps contemporain un temps historique et un temps «mythique». Les références artistiques stratifient votre écriture de manière étourdissante. C'est donc dire que vous organisez tous ces personnages dans un ensemble, bien sûr cohérent, mais surtout singulier et très signé. Vous avez déjà parlé d'un chœur et l'avez même métaphorisé à quelques reprises (par exemple dans les Manuscrits de Pauline Archange). Mais dans les romans depuis Soifs, il s'agit d'un chœur dirigé par un regard qui transcende tous les personnages, sans jamais s'autoriser un quelconque point de fuite (pas d'a parte du narrateur; rien que de la pensée et du discours rapportés). Il y a donc une harmonie, une sorte de timbre général qui empêche les voix d'être discordantes les unes par rapport aux autres, même si les idées émises par les personnages diffèrent et divergent. Les voix sont, quant à elles, parfaitement accordées, un peu comme à l'opéra où les conflits des personnages ne mettent jamais en péril la musique. Que permet, ou ne permet pas, cet emportement dans un flot général, au point de vue stylistique? Il y a un chœur, mais il y a aussi comme un seul corps vocal. Quelle portée a cette écriture «symphonique»?

MARIE-CLAIRE BLAIS Avant l'écriture du flot général, du rassemblement du chœur, si on peut dire, il me semble entendre une à une les voix des uns et des autres: si ce sont les voix des réfugiés cubains, tels que Julio et sa mère, le rythme est celui des vagues de l'eau cruelle qui emportera les enfants Ramon et Oreste. Il y a de nombreuses voix, mais chacune est différente de l'autre: Julio parle toujours en souvenir de sa famille perdue en mer et le ton est nostalgique, sinon d'une tristesse absolue ; la voix de Mère est plus affirmée, s'incarnant dans un présent que l'on peut voir (surtout dans le premier livre de Soifs, moins dans Mai au bal des prédateurs, où sa voix tout doucement semble se dissoudre en images éparses). Il faut quand vient l'écriture que toutes ces voix qui s'entrechoquent forment un tissage sonore uni. C'est un peu comme si vous étiez au théâtre et entendiez les acteurs dialoguer entre eux : qu'ils se voient ou non, il y a échange, comme il y a dans l'existence, même si nous sommes souvent incapables de le percevoir, un échange d'un être à un autre, parfois à travers la distance, car nous obéissons tous aux mêmes lois, de vie, de survie, dans un monde dont nous connaissons bien les beautés mais aussi les dangers. Dans les livres de Soifs, cela devient comme un chant de rumeurs, mais à travers ce chant suspendu à la réalité de chacun, il faut que les caractères des gens soient bien dessinés, leur fonction personnelle, leur identité, etc. J'espère répondre un peu à votre question.

VoIX ET IMAGES Votre réponse éclaire, d'une certaine façon, notre expérience de lecture. On dit souvent que, dans les romans du cycle Soifs, il n'y a pas de personnages principaux, que toutes les voix se confondent dans ce grand chœur. Mais ce n'est pas tout à fait juste. Et pour rendre compte de notre impression que certains personnages ont tout de même un rôle plus important dans tel ou tel volet, nous pourrions parler de ces voix qu'on entend au-dessus des autres. Ce que vous appelez très justement ce «tissage sonore uni » répond-il, dans votre sensibilité, à un sentiment d'harmonie qui vous fait opter pour cette fluidité? Comme si, au regard de la narration, l'humanité 
aspirait, malgré sa disparité et ses conflits, à un même "bien » ou bien-être. Cette question est un peu plus métaphysique que les précédentes, mais nous croyons qu'elle n'est pas du tout dissociée de la texture des derniers romans.

MARIE-CLAIRE BLAIS Oui, avec la fluidité du chœur dans ce cycle de Soifs, il y a de part et d'autre (que ce soit chez le juge Claude, le mari de Renata, ou chez un enfant qui sera jugé au tribunal, comme l'enfant Nathanael) une aspiration à la cohérence, à ce que vous appelez un "sentiment d'harmonie». C'est que tous sont des êtres humains rassemblés dans un même univers, lequel est toujours en évolution et en métamorphoses. Il y a cet espoir d'une évolution durable qui unit les uns aux autres, même à travers la séparation des classes sociales, la richesse ou la pauvreté, car on vit dans un monde où tout est vu et su, et assimilé par la conscience, et cela ne peut pas ne pas nous toucher de façon irréversible. Mais dans ces livres, on voit que l'aspiration à ce bien-être est aussi constamment compromise : les uns sont sauvagement piétinés pendant que d'autres continuent leur ascension matérielle. Comme on peut le constater dans la vie réelle, il y a la violence qui est toujours là, sourde, vengeresse, comme celle de Lazaro (laquelle devient criminelle dans Mai au bal des prédateurs); il y a malgré les efforts positifs de quelques-uns toujours cette régression prête à surgir - Mélanie et sa mère sont conscientes de ces puissances malsaines qui rôdent autour de leur maison (le racisme, etc.) et le sénateur noir à la retraite, Olivier (le mari de Tchouan et le père de Jermaine), que l'on retrouve dans tous les livres de Soifs, en retrace toute l'histoire dans ses articles, au point d'en éprouver le cauchemar à nouveau. C'est que le passé n'est jamais si loin du présent, que les erreurs du passé souvent empoisonnent le présent.

VoIX ET IMAGES Plus on avance dans cet entretien et plus une chose paraît évidente : vos personnages vous habitent, ils ont pour vous une existence presque tangible, à tel point que, d'une certaine manière, les stratégies esthétiques ne semblent servir que de support; la forme est celle qui permet le mieux d'entendre et de faire entendre toutes ces voix. Nous aimerions parler un peu de la façon dont vous construisez vos personnages. Vous dites, dans un entretien récent avec Janine Ricouart, que Renata est une « étude de plusieurs âmes que [vous avez] décrites en une seule avec plusieurs facettes ${ }^{4} »$. Cela rejoint une remarque que vous avez faite à Janieta Eyre il y a une dizaine d'années. Vous avez dit, de manière assez amusante: "I take real people and cocktail them ${ }^{5}$. " Cela fait penser à ce que Balzac a écrit dans l'avant-propos de $L a$ comédie humaine sur les types qu'il cherche à élaborer dans ses études de mœurs, à savoir qu'il s'agit justement d'une sorte de condensation de traits empruntés à plusieurs personnes. On sait que Balzac cherchait à faire l'histoire des mœurs de la société française, et que chaque type devait incarner une position, un rôle social, une vision du monde conditionnée par l'enracinement dans un milieu. Or, vos personnages semblent tenir d'une certaine façon (bien que complètement différente de celle de

4 Janine Ricouart, «Poète et politique : entretien avec Marie-Claire Blais », Janine Ricouart et Roseanna Dufault (dir.), Visions poétiques de Marie-Claire Blais, Montréal, Les Éditions du remue-ménage, 2008, p. 28.5 Janieta Eyre, "Tendencies and Philosophies. Marie-Claire Blais», The Power to Bend Spoons. Interviews With Canadian Novelists, Toronto, Mercury Press, 1998, p. 28. Traduction libre: Je prends des gens réels et je les mixe. 
Balzac) du type. Parfois on a le sentiment qu'ils incarnent une idée ou qu'ils représentent un discours qu'on peut reconnaître en tant que lecteur contemporain. Peuton penser que vous cherchez, un peu à la manière de Balzac, à faire le tableau des mœurs de votre époque? Le cycle Soifs, c'est peut-être un peu une Comédie humaine contemporaine, au fond?

MARIE-CLAIRE BLAIS C'est un portrait un peu impressionniste de notre époque, mais où tout frémit, bouge, change beaucoup, à travers les livres, mais un réel solide est en dessous de tout cela, avec des traits humains, sociaux, politiques, très reconnaissables (ou vraisemblables) autour d'une famille, d'une communauté de notre temps. Renata est une femme que l'on pourrait rencontrer dans le domaine de la justice, son mari aussi, sa beauté extérieure a pu appartenir à une vraie personne parmi mes connaissances, ici aux États-Unis, sa beauté intérieure aussi, mais le portrait a été transformé par des traits de caractère appartenant à d'autres êtres, lesquels rejoignaient l'esprit indépendant de Renata, sa recherche de justice pour les femmes criminalisées. Ce qui compte, c'est qu'il y ait là une vérité, une pensée proche de la nôtre aujourd'hui, avec nos actuelles préoccupations. Lazaro n'est pas que Lazaro: dès qu'il entre en livrant des huitres pour le banquet dans la maison d'Olivier et Tchouan, on peut sentir qu'il est d'un monde étranger et différent, qu'il refuse d'être un subalterne, et que son père égyptien, qui battait sa mère, est en lui, qu'il fut peut-être battu par ce père lui aussi; à cet instant, il n'est qu'un enfant humilié, il ne deviendra terroriste que beaucoup plus tard. Julio, que l'on voit dans le premier livre de Soifs être recueilli sur la plage, comme naufragé, et puis sauvé par la famille de Mère, se transformera au cours des années en un sauveur pour d'autres naufragés cubains et haïtiens, au point de devenir le directeur d'une maison-refuge. Ces réalités sont exactes, font partie de notre société à la fois destructrice et capable de grandes générosités insoupçonnables.

VoIX ET IMAGES Vos romans sont certainement une fresque du monde qui nous entoure dans sa diversité et sa complexité. Vous en faites en quelque sorte un tableau impressionniste où les personnages, leurs traits de caractère, leurs expériences, leurs préoccupations, dépendent de l'attention minutieuse que vous portez aux discours de la société, aux événements du monde et aux êtres que vous côtoyez. Mais votre écriture a ceci de particulier qu'elle semble chercher à capter ce portrait de la société depuis l'intérieur des consciences qui la peuplent, pour ainsi dire, en mettant en évidence, grâce à la forme, les liens qui nous unissent, et en «décrivant» ainsi une sorte de grande conscience supérieure, qui serait celle de notre temps. Par ailleurs, dans vos textes, l'art et l'artiste, qu'il soit écrivain, chorégraphe, musicien, photographe, peintre ou sculpteur, occupent une place centrale. Nombre de vos personnages insistent sur le caractère potentiellement transfigurateur de l'art, et vos textes sont tissés de références artistiques et intertextuelles qui colorent la manière dont vos personnages appréhendent le monde. On peut en déduire sans peine que le souvenir de vos lectures et votre expérience de l'art sous toutes ses formes colorent à leur tour votre écriture. Peut-on penser que l'élaboration de vos personnages dépend non seulement d'une observation fine du monde qui vous entoure, mais également du souvenir de vos lectures? Y a-t-il des personnages littéraires que vous retenez tout particulièrement et qui pourraient intervenir dans la construction de vos propres personnages, informer leur représentation? 
MARIE-CLAIRE BLAIS Oui, tous les écrivains sont perméables à leurs souvenirs de lecture, lesquels foisonnent sans cesse dans leur esprit: c'est un peu, cet attachement pour les œuvres qui nous ont nourris, emportés, un attachement semblable à celui du romancier pour ses personnages. Dans une conférence récente pour le Writers Union (Margaret Lawrence Conference, 2010), je tentais d'exprimer cet attachement très visible entre plusieurs auteurs et leurs personnages, ou héros, héroïnes : ainsi Truman Capote, si ému par le destin de ces deux jeunes criminels (In Cold Blood) qu'il croyait pouvoir dépeindre avec distance, ou tant d'autres, Faulkner et cette tendresse que l'on peut sentir pour Temple, l'étudiante de Sanctuary - plus encore peut-être pour Joe Christmas, le jeune assassin noir de sa maîtresse blanche, et qui finira par être pendu, dans Light in August. Pour ces auteurs, Temple, le petit Joe Christmas au sinistre passé, ce sont de vraies personnes qu'ils semblent avoir intimement connues ; pour nous aussi, il en est de même, notre attachement devient tout aussi profond et éternel, pour les personnages de Proust, de Virginia Woolf, tous nous inspirent, et on ne cesse de les analyser, d'analyser leurs motifs, les motifs de ces deux jeunes meurtriers dans In Cold Blood ou celui du jeune homme dans Crime et châtiment. C'est aussi pour l'écrivain, cette passion, un grand moyen de connaissance.

VoIX ET IMAGES Votre réponse est surprenante, mais peut-être d'autant plus éclairante. Dans le cycle Soifs, il y a bien sûr de nombreux récits de jeunes meurtriers, de mères infanticides, et puis il y a Lazaro, celui qui devient terroriste. Vos personnages s'acharnent à essayer de comprendre comment ces meurtriers ont pu en arriver là et à analyser leurs motifs. Mais nous ne croyions pas que tous ces personnages criminels étaient «nés » aussi d'une filiation littéraire. Nous ne les avions jamais envisagés, pour ainsi dire, comme des descendants de Raskolnikov (par exemple).

MARIE-CLAIRE BLAIS C'est intéressant, ce que vous exprimez sur les descendants de Raskolnikov, mais sans doute que Raskolnikov fut pour son auteur un vrai jeune homme meurtrier dont il dut apprendre l'histoire à travers les journaux ou de façon semblable; le grand maître de l'écriture russe le sortirait de l'ombre, sortirait de l'ombre aussi tant de misère sociale liée à ce meurtre. Si bien que cette histoire deviendrait universelle. Je connaissais sans doute l'existence de Raskolnikov au moment où j'écrivais L'exécution ${ }^{6}$. Dans cette pièce de théâtre, Kent pourrait se rapprocher de Raskolnikov pour la gratuité du meurtre, mais ce crime collectif (à la fin, collectif) avait vraiment eu lieu dans un collège au Québec, dans les années 1960; j'ai poursuivi cette étude de Kent, l'auteur du meurtre du petit Éric, dans d'autres livres, telle la trilogie des Manuscrits de Pauline Archange, à travers le jeune homme accusé du meurtre de son père juge, Philippe L'Heureux. Le second meurtrier, Stéphane, est celui qui portera le meurtre dans L'exécution, celui qui en sera l'inguérissable blessure de conscience.

VOIX ET IMAGES On a souvent commenté votre œuvre romanesque en y soulignant la part faite aux souffrances des marginaux, des exclus, des réprouvés. Vous déclarez vous-même vouloir transmettre une certaine grâce par l'esthétique, susciter des

\section{$+++$}

6 L'exécution. Pièce en deux actes, Montréal, Éditions du Jour, 1968, 118 p. 
sentiments généreux et courageux envers l'humanité. La dimension éthique de votre écriture est en effet très tangible. Mais votre œuvre est aussi complexe, ne s'octroie pas si facilement le pouvoir de décider du bien et du mal. Les figures de pouvoir et d'autorité, les personnages appartenant aux classes privilégiées ont manifestement droit à la même empathie que les marginaux, au sein de votre écriture, qui procède par vagues, n'épargnant ni n'oubliant personne. Si la compassion est certainement la tonalité la plus prégnante de votre œuvre, le registre pulsionnel du désir, de sa violence, ne laisse aucune créature intacte, et la voix qui les décrit de l'intérieur mais toujours à la troisième personne est elle-même emportée par le rythme des butées qui les révèlent tous insatiables, obstinés, assoiffés. Nous aimerions que vous nous en disiez plus sur les pouvoirs de cette omniscience que vous avez réinventée et qui produit à la lecture une vue d'ensemble à la fois fragmentée, «insulaire » et universellement irradiante. Est-ce juste de dire que cette position vous permet de ne pas trancher trop vite entre victimes et bourreaux?

MARIE-CLAIRE BLAIS Comme auteur, on ne peut trancher entre victime et bourreau : il faut entrer dans l'âme de chacun. C'est un peu ce qui se passe avec Renata lorsqu'elle devient juge dans les livres de Soifs et qu'elle doit décider du sort de femmes criminelles: elle cherche à comprendre, va jusqu'à la source du mal subi - dans le cas de ces prisonnières, il y aura souvent un viol, une maltraitance violente de ces femmes au tout début de leur vie.

VoIX ET IMAGES Il y aurait, dans le cocktail que constituent vos personnages, l'attention portée aux êtres qui vous entourent dont vous prélevez ici et là des traits, le souvenir de vos lectures, ainsi que le souvenir de personnages plus anciens. Dans le personnage de Mère, par exemple, il pourrait y avoir des échos de la Grand-Mère Antoinette, et Vincent, c'est peut-être un peu Emmanuel, dans un tout autre contexte. Mais laissons là cette discussion sur les personnages, car nous aimerions parler justement du contexte ou, plus précisément, du choix de lieu. Les États-Unis, où vous vivez, semblent être pour vous le réceptacle du temps historique contemporain. Les lieux, les villes où éclatent les violences sociales sont nommés, repérables. L'espace québécois a quant à lui souvent connu un traitement plus estompé, plus mythique dans vos romans. Et il n'existe à peu près plus dans les derniers romans. Cette géographie singulière, américaine, que vous recomposez dans vos livres est-elle la seule qui puisse capter un certain temps historique? Ou est-ce une simple contingence qui vous fait puiser directement dans ce qui vous entoure? Les violences symboliques et sociales beaucoup moins apparentes mais tout de même très présentes au Québec vous semblent-elles impossibles à traiter?

MARIE-CLAIRE BLAIS Les violences sociales du Québec peuvent être traitées à travers les autres, les universelles, d'une manière qui englobe d'autres sociétés que la nôtre mais qui lui sont proches; je crois que, dans la trilogie des Manuscrits de Pauline Archange, ces sujets étaient traités très directement - à travers une écriture lyrique, mais très directement quand même. Je ne dirais pas que ce traitement de l'injustice, ou du sentiment d'injustice que subissent beaucoup d'êtres, est désormais un traitement «estompé» dans mes livres. Les thèmes se sont élargis, oui : ils sont abordés à travers la société américaine, mais aussi en dehors, comme avec le pèlerinage que fait le moine Asoka dans le monde, de Moscou à l'Inde, etc. ; de même on voit dans 
Naissance de Rebecca à l'ère des tourments Ari, le père de la petite Lou, suivre le moine en Amérique centrale, etc. Je pense à un livre écrit beaucoup plus tôt, David Sterne, récemment réédité en poche au Boréal, qui pourrait être situé dans un Québec moderne - il y est question de la guerre du Vietnam, qui a préoccupé les États-Unis, mais aussi toute l'Amérique, le monde entier: David Sterne, par la révolte de sa jeunesse, pourrait être un héros québécois, de même que Pierre, car les groupes de motocyclistes dont je me suis inspirée existaient déjà au Québec comme aux États-Unis.

VoIX ET IMAGES La question vise en fait la manière dont vous représentez l'espace, le lieu géographique dans votre écriture; ou plus précisément l'imaginaire particulier de l'espace (géographique et politique) et son rapport avec l'histoire. Que les violences sociales du Québec puissent être traitées à travers les autres est certain, et que vous en ayez traité de manière très directe dans vos romans antérieurs ne fait pas de doute. Mais vous ne représentez pas l'espace québécois et l'espace américain de la même façon; c'est d'abord l'espace (québécois) et non les violences sociales qui reçoit un traitement plus mythique, plus "estompé». Du moins, c'est l'impression que nous avons à la lecture. Les lieux, les événements qui y sont associés sont souvent nommés de façon très explicite dans vos romans "américains»; ils semblent même participer de la dimension incantatoire de la narration. C'est moins le cas dans les autres livres. Avec Le sourd dans la ville, vous entreprenez un travail du texte qui parvient à son point d'aboutissement dans le cycle Soifs, et c'est dans l'espace américain que ce concert de voix atteint sa plus grande ampleur. Ce que nous aimerions, donc, c'est comprendre comment l'écrivaine que vous êtes trouve son matériau, ses images, son phrasé dans des inscriptions qui sont typiquement américaines justement: la guerre du Vietnam (qui a préoccupé tout le monde mais qui a engagé seulement les Américains), la ségrégation ou la libération des noirs, la peine de mort. L'universel n'est rejoint que par le particulier, or c'est ce particulier (universel) qui nous intéresse: le contexte américain, sa singularité, permet-il de mieux accéder aux grandes questions sociales, et si oui, dans quelle mesure?

MARIE-CLAIRE BLAIS Selon moi, ces sujets soulevés dans ce cycle de Soifs nous concernent tous : la ségrégation, le racisme, la peine de mort (nous avions au Canada la peine de mort il n'y a pas si longtemps, il ne faut pas l'oublier; quant au racisme, nous avons eu bien des preuves que cela existe aussi au Québec), tous ces maux, ces fléaux ne sont-ils pas toujours latents partout? Voyons comment on traite les gitans, en ce moment, en France, en les chassant du pays de façon impitoyable. Ce sont des maux universels, d'universelles intolérances qu'il faut absolument dénoncer. Mon approche américaine a eu lieu la première fois quand je me suis retrouvée seule à Cambridge à 23 ans, à une époque où tout allait être bouleversé, transformé, dans ce pays. Je crois que ces premières années (près de sept ans la première fois) m'ont profondément marquée parce que je voyais des gens de ma génération agir de façon positive afin que les lois ne soient plus ségrégationnistes, quand naissait aussi un grand mouvement de non-violence, un désir de paix immense et partagé, et que je côtoyais des êtres extraordinaires qui, vraiment, changeraient l'état du monde. Je pense que déjà je portais ce cycle de Soifs mais ne pouvais encore décrire ce que je ressentais, puisque j'écrivais alors Une saison dans la vie d'Emmanuel, les Manuscrits de Pauline Archange et en même temps un livre plus américain, David Sterne. 
VoIX ET IMAGES La géographie ne semble cependant jamais indifférente. Par exemple, l'île du cycle Soifs, parce qu'elle renferme une si grande diversité (d'ethnies, de classes et de situations sociales, etc.) dans un espace restreint, agit comme un microcosme. En conjuguant l'espace et la mémoire de cette galerie de personnages, vous parvenez à faire un portrait universel. Et il y a peut-être des particularités de l'espace américain qui permettent, voire favorisent ce genre de représentation. Avec Mai au bal des prédateurs, on a tout à la fois l'impression que certaines boucles sont bouclées et que d'autres pistes s'ouvrent, notamment avec l'apparition de Yinn et de sa bande de filles. Or, il me semble qu'on retrouve dans ce cinquième volet un écho des deux rassemblements simultanés qui se déroulaient dans Soifs. Dans le premier volet, il s'agissait bien sûr de souligner l'aube d'un nouveau millénaire et la naissance de Vincent. Malgré une hantise apocalyptique persistante, l'accent était mis sur la naissance et les recommencements. Dans le cinquième volet du cycle, l'accent est mis sur la mort imminente, sur le masque, le spectre, l'évanescence. On peut penser au défilé des filles condamnées orchestré par Yinn et Hermann et à cet autre "défilé», celui des fantômes de Mère et des images fugaces de sa conscience vacillante. Pouvez-vous commenter le rôle que joue ce cinquième volet dans le cycle? Nous croyons que celuici ne s'achèvera pas ainsi...

MARIE-CLAIRE BLAIS Bien sûr, le cycle ne s'achèvera pas ainsi, il est toujours en mouvement vers un autre livre et quelque chose de nouveau, chaque fois, mais bien des personnages précédents seront encore là aussi, dans le sixième livre, dans le déroulement de leurs propres vies, qui continuent, qui changent aussi. Le défilé des travestis, dans Mai au bal des prédateurs, avec le spectre de la mort imminente, est une sorte de défilé mondial (Yinn se réfère constamment à l'Afrique quand il voit le défilé au-delà des océans, Fatalité se compare aussi à une victime du sida africaine et cela revient sans cesse : le défilé se passe en même temps partout). J'ai bien hâte que l'on saisisse cela, qui est essentiel. Fatalité, qui vit en Amérique du Nord, n'a pas d'argent pour acheter les médicaments qui le sauveraient, comme tant d'autres personnes comme lui, partout dans le monde. La jeune Africaine Rosannah non plus ne peut se procurer ces médicaments. Plus que sur la mort imminente (car ces jeunes gens auraient pu être sauvés), l'accent de détresse est mis sur la gravité de cette maladie que l'on feint d'ignorer, comme la famine ou la guerre, en se disant qu'elle peut être guérie. Aussi, comme pour Caroline dans les livres précédant Mai au bal des prédateurs, la mort ne sera jamais présentée comme une mort réelle, ou une fin : c'est une sorte de recherche des sensations de la vie jusqu'au bout, et un cheminement dans une vaste mémoire où Mère se réconcilie avec tous les siens, cherche à mieux les décrire et les comprendre. Déjà elle parle de ses lettres à Augustino, déjà c'est la continuité, comme la vie continue avec les photographies de Caroline, les rêves que les autres font à son sujet, comme si la frontière entre vie et mort existait à peine, n'était qu'un faux miroir. Il y a Mai et ses frères qui sont tous si jeunes et débordants de vie, Yinn et ses amis tous très vivants, et artistes, Petites Cendres qui est fragile mais déborde aussi de vie, de passion; il y a ce fleuve ou cette mer ou cet océan de vies qui fusionnent dans un même élan, une indescriptible vitalité, et la mort qui est là pour chaque être vivant. Mais dans ce livre, ce n'est pas là ce qui compte, c'est plutôt l'espoir de l'amour, la compréhension, et aussi une sorte de grande beauté qui est toujours là. 